\title{
Microdissection and microcloning of chromosomal alterations in human breast cancer
}

\author{
Jeffrey M. Trent ${ }^{1}$, Barbara Weber ${ }^{2}$, X.Y. Guan ${ }^{1}$, Ji Zhang ${ }^{1}$, Francis Collins ${ }^{1}$, Ken Abel ${ }^{2}$, Austin Diamond ${ }^{2}$, \\ and Paul Meltzer ${ }^{1}$ \\ ${ }^{l}$ Laboratory of Cancer Genetics, National Center for Genome Research, National Institutes of Health, \\ Bethesda MD, USA; ${ }^{2}$ Department of Internal Medicine and the Human Genome Center, University of \\ Michigan, Ann Arbor MI, USA
}

Key words: chromosomes, breast cancer, chromosome microdissection, physical mapping

\section{Summary}

The recognition of recurring sites of chromosome changes in malignancies has greatly facilitated the identification of genes implicated in the pathogenesis of human cancers. Based especially upon recent studies [1-4], it appears increasingly likely that a subset of recurring chromosome alterations will be recognized in human breast cancer. Currently recognized chromosome changes characterizing breast carcinoma include the recognition of cytologic features of gene amplification (e.g. double minutes [dmins] and homogeneously staining regions [HSRs]) [5-8]. As these and other chromosome regions are implicated in recurring abnormalities in breast cancer, it will become increasingly important to have bandor region-specific genomic libraries and probes in order to facilitate high resolution physical mapping and ultimately to clone breast cancer related genes [9]. Toward this end an important recent development in physical mapping has been the establishment of chromosome microdissection as a rapid and reproducible approach to rapidly isolate and characterize chromosome region-specific DNA, greatly facilitating the initial steps in positional cloning of disease-related genes [10-13]. In this brief report, we will highlight the application of chromosome microdissection to the generation of region-specific probes for both fluorescent in situ hybridization (FISH) and the generation of genomic microclone libraries. Additionally, efforts using this methodology to generate a microclone library encompassing the early onset breast/ovarian cancer (BRCA1) gene will be presented.

Presented by Jeffrey M. Trent at the 16th Annual San Antonio Breast Cancer Symposium, San Antonio TX, USA, November 4, 1993; Minisymposium on "Molecular Genetics in Breast Cancer".

Address for correspondence and offprints: Jeffrey M. Trent, Laboratory of Cancer Genetics, National Center for Genome Research, National Institutes of Health, 9000 Rockville Pike, Bldg 49 Rm 4A22, Bethesda MD 20892, USA; Tel: $301-402-$ 2023; Fax: 301-402-2040 


\section{Generation of chromosome and band region- specific probes by chromosome micro- dissection: Application to chromosome alterations in malignancies}

For many human malignancies, the analysis of cytogenetic alterations has become an essential component of the diagnostic and prognostic workup of the patient (for review see [14]). Careful examination of recurring chromosome alterations in a variety of cancers has led to the identification of recurring (specific) sites of chromosome change which has in turn led to the stratification of patients by chromosome abnormality into defined subgroups. Following systematic testing of these genetic alterations as prognostic factors, results for many cancers indicate that chromosome change represents an important (and often independent) variable capable of predicting duration of remission and survival [14].

Unfortunately, despite significant recent gains, our knowledge of chromosome alterations in most solid tumors (including human breast cancer) is limited [15], and has been complicated by numerous factors. One of the most significant technical limitations of conventional cytogenetic techniques is the inability to characterize unequivocally all cytologically recognizable chromosome rearrangements. Accordingly, the presence of "marker" chromosomes (whose chromosomal origin is unknown) and the recent identification of unidentifiable "cryptic" translocations [16] has caused even the most comprehensive search for chromosome rearrangements to be incomplete in its characterization of most cancers.

One recent development which has been of significant assistance in visualizing chromosome alterations in malignancies has been the introduction of whole chromosome composite "painting" probes (WCPs) for use in fluorescent in situ hybridization (FISH) [17]. Unfortunately, using this approach to characterize unknown chromosome rearrangements requires access to all 24 chromosome painting probes, and even when positive information is obtained it fails to sublocalize the change to a specific band-region.
Our laboratory recently described a procedure (termed "Micro-FISH") [10] which allows the rapid generation of chromosome and region specific probes by combining microdissection and chromosome painting. This approach has led to the direct identification of chromosome alterations in several malignancies, including the routine identification of changes unidentifiable by standard chromosome banding $[10,12,13,18]$. Figure 1 illustrates the utilization of whole chromosome Micro-FISH probes when applied to either normal or tumor metaphases. This approach allows the identification of translocations, insertions, and other structural rearrangements of chromosomal material. Figure 2 illustrates the utilization of chromosome microdissection to generate band region-specific probes which provide useful probes for metaphase chromosomes and potentially for application to interphase nuclei $[10,19]$.

The utility of microdissection in the analysis of human breast cancer is closely tied to the study of chromosome alterations in breast cancer by banding analysis. Recently our laboratory reported the description of chromosome alterations in 62 cases of breast cancer -28 cases of primary tumors and 34 cases of metastatic lesions [3,4]. Figure 3 provides pictorial documentation of the distribution of structural chromosome alterations in both primary and metastatic breast carcinomas. The combined information from this series of cases implicated several chromosomes, but most notably chromosomes 1,6 , and 16 in primary cancers, and 1,7,11, and 6 in metastatic breast cancers (the reader is referred to references $3 \&$ 4 for specific information on chromosome breakpoints). The application of this information is that Micro-FISH probes can now be generated for the regions showing the highest frequency of rearrangement and applied to cases where even routine cytogenetic information is indeterminate due to poor technical quality of mitoses. Utilizing this information, it is hoped that further insights into the frequency and specificity of regions most consistently altered in human breast cancer will be obtained. 

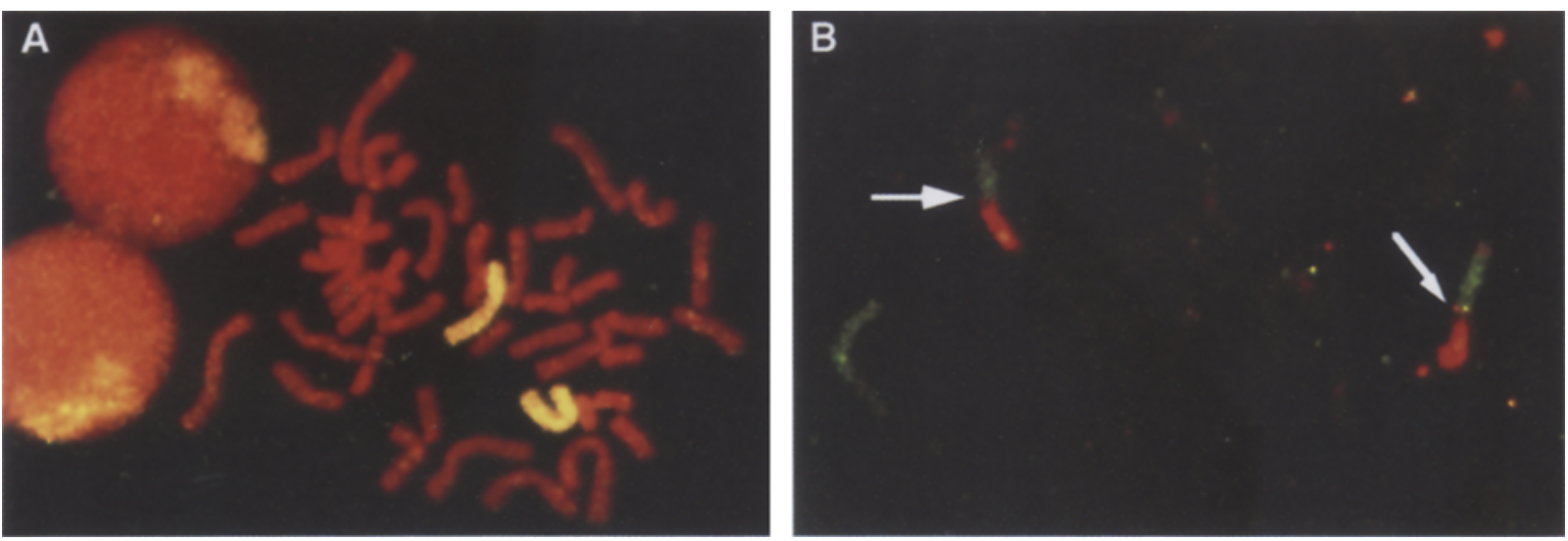

Figure 1. Generation and utilization of whole chromosome painting probes (WCPs) by chromosome microdissection and their application in identifying chromosome rearrangements. A) WCP for human chromosome 6 hybridized to normal mitoses, documenting the intense and uniform hybridization of microdissection generated WCPs [19]. B) The application of microdissection WCPs on tumor mitoses to identify chromosomal rearrangements in tumor mitoses. The mitoses shown here demonstrate the utilization of WCPs for chromosomes 3 (green) and 13 (red) simultaneously FISHed to a tumor cell displaying a nonreciprocal $t(3 ; 13)$ translocation (arrows).

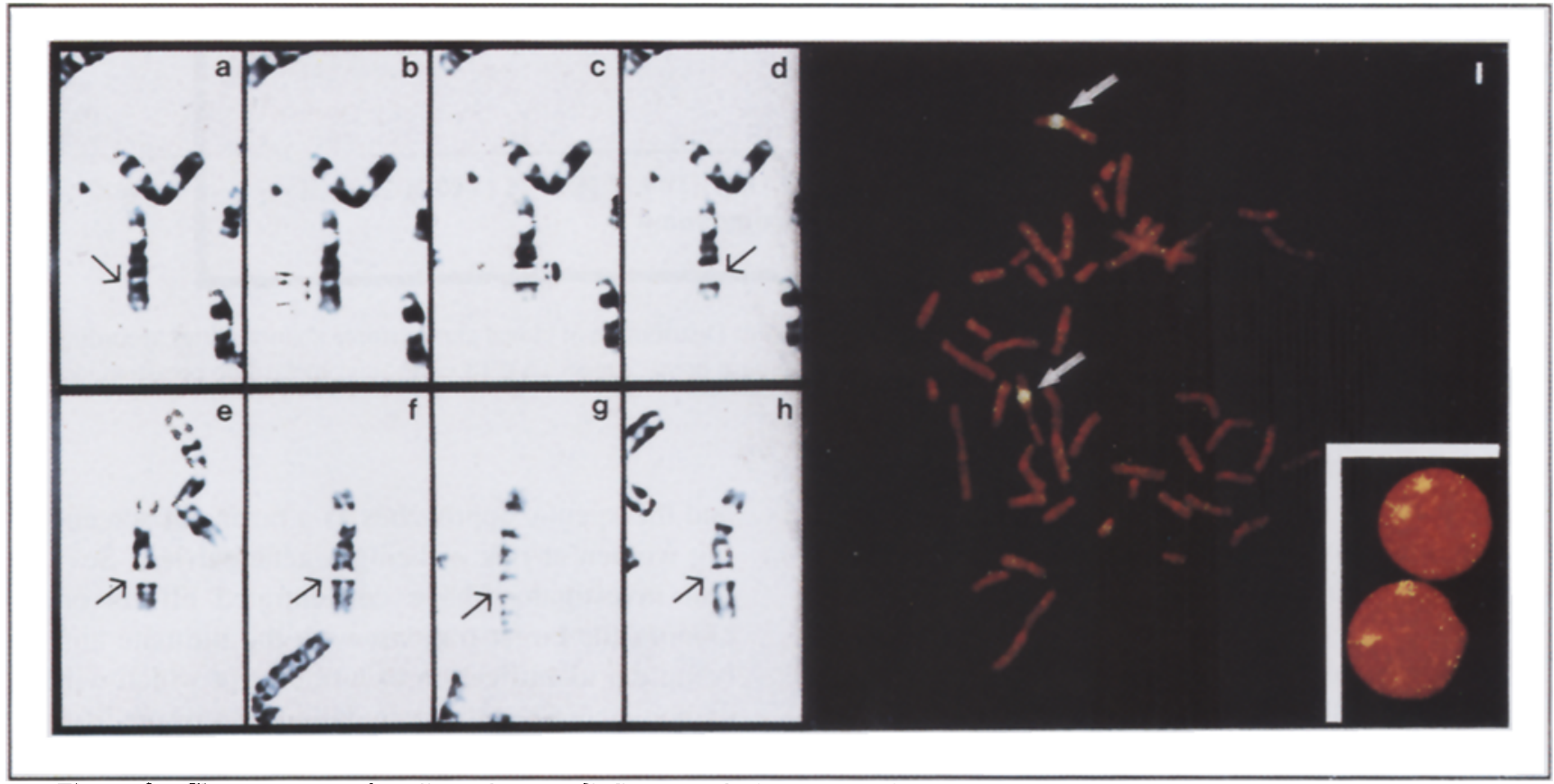

Figure 2. Chromosome microdissection. a-d) Sequential photographs illustrating the microdissection of chromosome band region 6q21-23. e-h) Series of dissected chromosome 6's to illustrate the reproducibility of dissection. i) A Micro-FISH probe for 6q21-23 was hybridized to a normal peripheral blood lymphocyte metaphase, demonstrating the band-specific fluorescence. The inset shows hybridization to the domains of interphase nuclei that encompass chromosome 6q21-23 (from reference 10, with permission). 


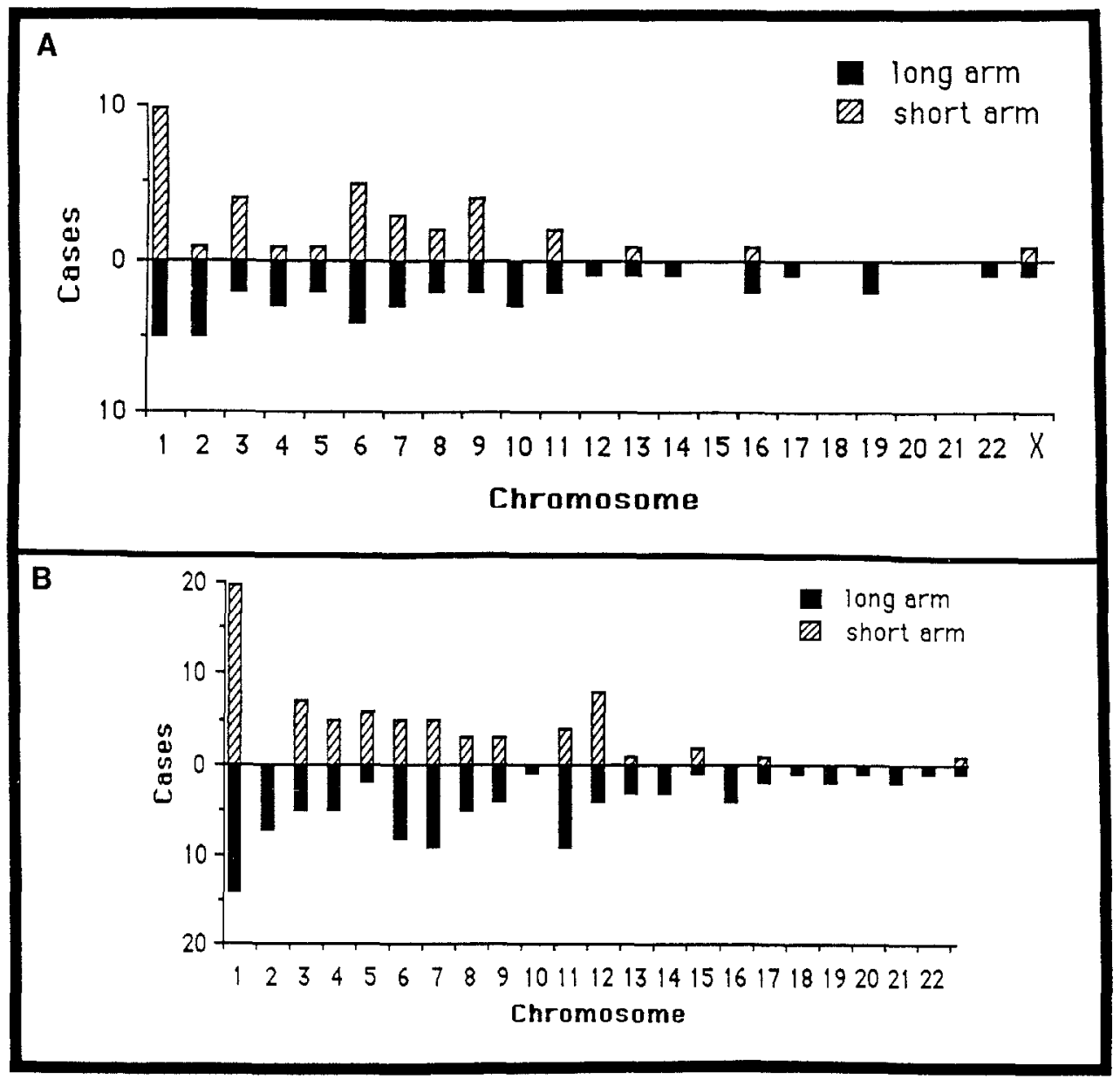

Figure 3. Clonal structural rearrangements in human breast cancer. Distribution of clonal chromosome abnormalities according to short and/or long arm of each human chromosome from primary breast cancer (A), 18 cases, and metastatic breast cancer (B), 32 cases (from references $3 \& 4$, with permission).

\section{Generation of chromosome band-specific} genomic libraries by chromosome microdissection: Application to the BRCA1 locus

As described in detail elsewhere in this volume [20], the q12-21 region of chromosome 17 is known by linkage analysis to contain a gene or genes conferring susceptibility to early-onset breast cancer and ovarian cancer (termed BRCA1). It is assumed that the identification and ultimately the molecular (mechanistic) characterization of this gene will provide enhanced understanding of the pathogenesis of breast cancer, and will lead to development of targeted diagnostic and therapeutic approaches as a means of screening women at risk of being a gene carrier. Several investigators have concentrated efforts on cloning the target regions, with the ultimate aim being the identification of a transcript which will demonstrate a hereditary mutation thereby qualifying as a candidate gene for this disorder. As described briefly below, the application of chromosome microdissection has played an important role in rapidly generating "entry point" clones within the BRCA1 region to augment other strategies for cloning this gene.

The framework for this analysis is the significant recent success in "positional cloning", which 
(after identification of a disease gene locus by linkage analysis) involves first the saturation of a candidate region with highly polymorphic markers, and subsequently generation of a physical map using cosmids, yeast artificial chromosomes (YACs), or other large insert clones [9]. The goal of positional cloning is to cover as quickly as possible the entire region of interest, and then begin to systematically search through transcripts of genes residing within the boundaries of the disease gene locus. This approach has now been successfully used to find such diverse disease genes as cystic fibrosis and neurofibromatosis, as well as several tumor suppressor loci (for review see [9]).

For the BRCA1 gene, linkage analysis and polymorphic markers have narrowed the candidate gene region to an interval encompassing 17q1221 , and proximal to an anonymous DNA marker termed MFD188 [20,21]. Microdissection of the $17 q 12-21$ region of a normal chromosome 17 was performed in our laboratory, followed by PCR amplification of the microdissected material (using a universal primer which allows amplification of a wide range of DNA sequences) and cloning of these PCR products into a modified pGEM vector. The BRCA1 microclone library contained approximately 100,000 recombinant "microclones" (average insert size -500 base pairs), and although the library has not been rigorously analyzed for redundancy, no identical clones were observed in the first 118 which have been analyzed for single copy sequences. The microclone DNA library was also labeled directly for FISH and hybridized to normal human chromosomes simultaneously with a fluorescently labeled cosmid derived from MFD188 (Figure 4). As demonstrated in this figure, the microdissected material is centered just centromeric to the BRCA1-linked marker MFD188, thereby demonstrating that our microclone library encompasses the BRCA1 region.

Of the first 118 individual microclones examined, 65 were shown to contain repetitive sequences when screened with $\left[{ }^{32} \mathrm{P}\right]$-labeled Cot-1 DNA. Clones with no evidence of hybridization to Cot-1 DNA were hybridized to a panel of DNAs by Southern blotting. Filters containing total human DNA, total mouse DNA, and DNA prepared from 7AE4 (a somatic cell hybrid containing human chromosome 17 as the only human DNA in a rat hepatoma cell background [22]) were examined. Using this method, several DNA microclones have been positively identified which map to human chromosome 17. Six of these microclones which met the criteria for containing only single-copy sequences have been sequenced for PCR primer design and analyzed for map location with a previously published radiation hybrid mapping panel for chromosome 17 [22]. Three of these microclones fell directly within the BRCA1 region, with the remaining three falling just outside the current flanking markers. The isolation of clones which fall within or just outside the BRCA1 region attests to the high degree of accuracy possible with marker-directed chromosome microdissection. Finally, a chromosome 17 cosmid library has been directly screened with the microdissected probe as well as with the pool of 6 clones known to reside within or near the BRCA1 locus. Both novel cosmids and hits on previously identified cosmids for this region were obtained, further defining the utility of this approach in the generation of overlapping clones (i.e. contig assembly) spanning the BRCA1 locus.

\section{Isolation of amplified DNA by chromosome microdissection: Application to human breast cancer}

A voluminous literature now exists detailing the frequency of gene amplification in human and experimental cancers (for review see ref. 5). Of relevance to this article, breast cancer has been shown to have among the highest frequency of gene amplification of any malignancy $[5,6]$. The genes most frequently amplified in breast cancer are HER-2/neu, INT-2, and c-myc [6]. To date, a large number of clinical trials have demonstrated the relevance of HER-2/neu amplification 


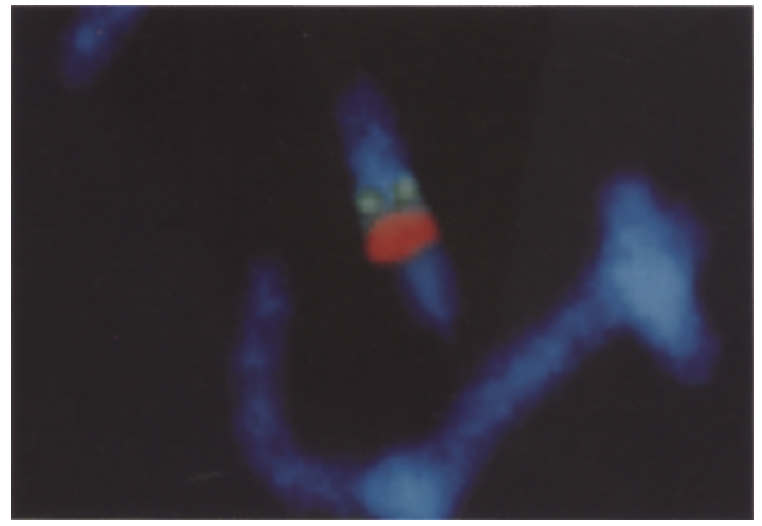

Figure 4. FISH documenting the regional localization of a BRCA1-DNA microclone library (labeled in red) relative to an anonymous DNA marker (MFDl88, labeled in green) known to be distal to the BRCA1 gene [20] [see text].

to breast cancer natural history [8]. The frequent occurrence of c-myc amplification likewise appears to be associated with a subset of breast cancer patients with a poor prognosis.

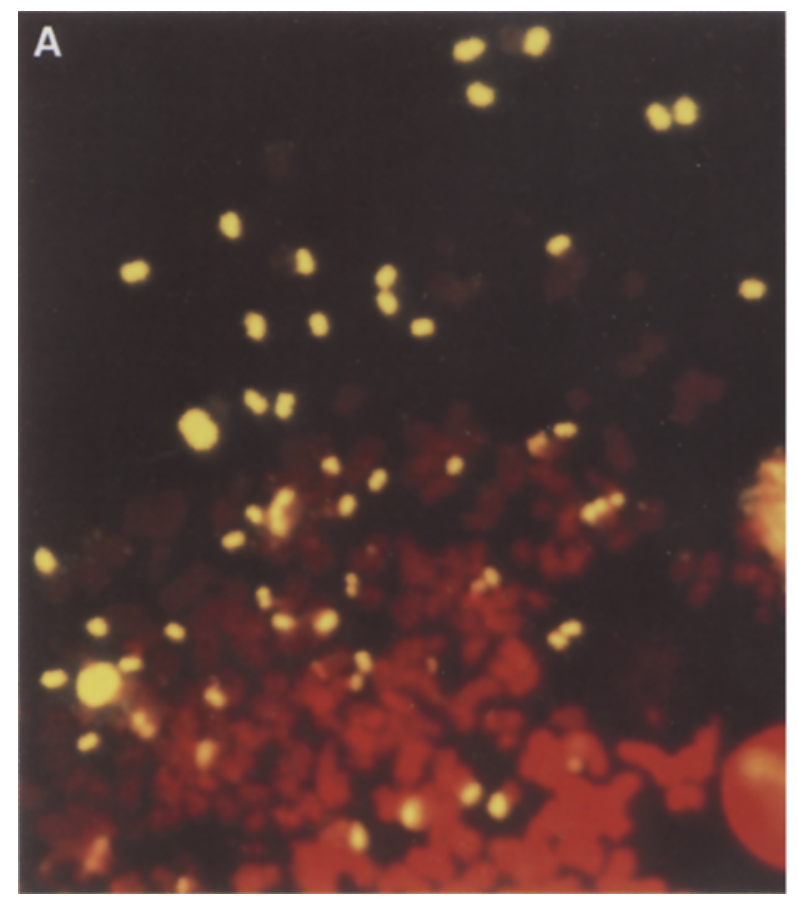

In addition to these studies indicating the amplification of known cellular oncogenes, very recent studies by the laboratory of Dr. Joe Gray and colleagues have indicated that unrecognized amplification units remain to be characterized in human breast cancer. This work was based upon the development of a procedure called Comparative Genome Hybridization (CGH), which takes advantage of the hybridization of fluorescently labeled tumor and normal DNA to normal metaphase chromosomes, highlighting regions of both gain (DNA sequence amplification) and loss [23]. The application of chromosome microdissection to this process has enabled our laboratory to provide the complement of this approach, which is to dissect cytologically recognizable manifestations of gene amplification (e.g. dmins and HSRs) from tumor cells and then to unequivocally determine the chromosomal composition of the amplified sequences (a procedure we call Hybridization for Genomic Content — HGC [24]).

Figure 5 provides an illustration of the cyto-

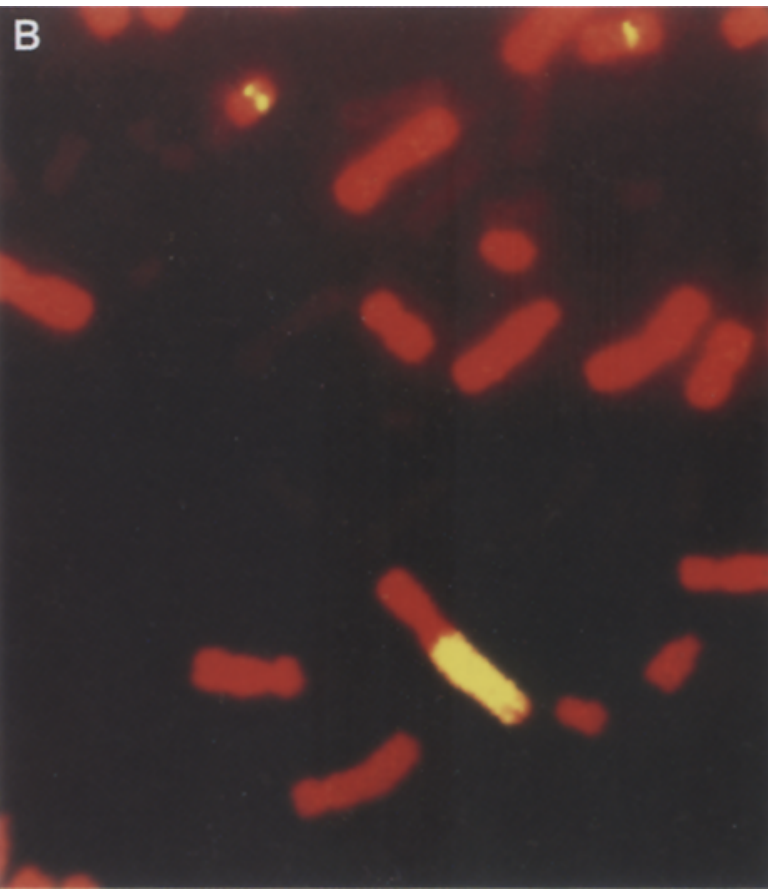

Figure 5. FISH documenting the isolation by chromosome microdissection of amplification unit probes which, following FISH, recognize cytogenetic evidence of gene amplification (in yellow) - A) double minutes; B) homogeneously staining region. 
logic representation of gene amplification from two tumors, one containing dmins and the other an HSR. In each case chromosome microdissection was utilized to target either the double minutes or the HSR, PCR-amplifying the dissected DNA and using this as a probe for FISH back to the original tumor mitoses. This procedure has resulted in the generation of probes which clearly highlight the amplified domain. It is then possible to FISH this amplification unit probe back to normal metaphases to determine precisely the chromosomal component of the amplified domain. Finally, as successfully modeled in another tumor type [13], this targeted microdissected PCR product can also be cloned as a rapid way to gain entry point clones for ultimate positional cloning and identification of genes amplified in human cancers.

\section{Overview and future perspectives on chromosome microdissection}

This study clearly demonstrates the potential of chromosome microdissection to facilitate the identification, cloning, and characterization of chromosomal rearrangements in human cancers. The strategy of using chromosome microdissection combines all steps for detecting, cloning, mapping, and ultimately isolating genes into a single process that is readily applicable to any cytologically recognizable chromosome rearrangement. The application of chromosome microdissection in human breast cancer may have its most immediate impact on the analysis of amplified domains, with the ability to dissect dmins or HSRs from any source of metaphase cells (including primary tumors and tumor cell lines), increasing the likelihood that new target genes will be found within amplification units. Ultimately, as recurring sites of chromosome change in breast cancer are identified, further targets for chromosome dissection will be addressed using this approach. It is hoped that these targeted microdissections will provide a valuable resource for studies aimed at mapping and isolating genes important in human breast cancer.

\section{References}

1. Hainsworth P, Raphael $\mathrm{K}$, Stilwell $\mathrm{R}$, Bennett $\mathrm{R}$, Garson O: Cytogenetic features of twenty-six primary breast cancers. Cancer Genet Cytogenet 52:205-218, 1991

2. Saint-Ruf C, Gerbault-Seureau M, Viegas-Pequignot E, Zafrani B, Malfoy B, Dutrillaux B: Recurrent homogeneously staining regions in $8 \mathrm{p} 1$ in breast cancer and lack of amplification of POLB, LHRH, and PLAT genes. Cancer Genet Cytogenet 52:27-35, 1991

3. Thompson F, Emerson J, Dalton W, Yang J, McGee $D$, Villar H, Knox S, Massey $K$, Weinstein $R$, Bhattacharyya A, Trent J: Clonal chromosome abnormalities in human breast carcinomas, 1: Twentyeight cases with primary disease. Genes Chrom Cancer 7:185-193, 1993

4. Trent J, Yang J, Emerson J, Dalton W, McGee D, Massey K, Thompson F, Villar H: Clonal chromosome abnormalities in human breast carcinomas, II: Thirty-four cases with metastatic disease. Genes Chrom Cancer 7:194-203, 1993

5. Kellems R (ed): Gene Amplification in Mammalian Cells. Marcel Dekker, New York, 1993

6. Alitalo K, Schwab M: Oncogene amplification in tumor cells. Adv Cancer Res 47:235-281, 1986

7. Slamon D, Clark G, Wong S, Levin W, Ullrich A, McGuire W: Human breast cancer: Correlation of relapse and survival with amplification of the HER-2/neu oncogene. Science 235:177-182, 1987

8. Wahl G, Carroll S, Windle B: Cytogenetic and molecular dynamics of mammalian gene amplification: Evidence supporting chromosome breakage as an initiating event. In: Kellems, $\mathbf{R}$ (ed) Gene Amplification in Mammalian Cells. Marcel Dekker, New York, 1993, pp 513-532

9. Collins F: Positional cloning. Nature Genetics 1:3-8, 1992

10. Meltzer P, Guan XY, Burgess A, Trent J: Rapid generation of region specific probes by chromosome microdissection and their application. Nature Genetics 1:24-28, 1992

11. Guan XY, Trent $J$, Meltzer P: Generation of bandspecific painting probes from a single microdissected chromosome. Human Mol Gen 2:1117-1121, 1993

12. Guan XY, Meltzer P, Cao $J$, Trent J: Rapid generation of region-specific genomic clones by chromosome microdissection: Isolation of DNA from a region 
frequently deleted in malignant melanoma. Genomics 14:680-684, 1992

13. Zhang J, Trent J, Meltzer P: Rapid isolation and characterization of amplified DNA by chromosome microdissection: Identification of IGF1 R amplification in malignant melanoma. Oncogene 8:2827-2831, 1993

14. Heim S, Mitelman F: Cancer Cytogenetics. Alan R. Liss, New York, 1987

15. Mitelman F: Catalog of Chromosome Aberrations in Cancer, 4th Edition. Wiley-Liss, New York, 1992

16. Meltzer $\mathrm{P}$, Guan X-Y, Trent J: Telomere capture stabilizes chromosome breakage. Nature Genetics 4:252-255, 1993

17. Pinkel D, Landegent J, Collins C, Fuscoe J, Segraves $\mathrm{R}$, Lucas $\mathbf{J}$, Gray J: Fluorescence in situ hybridization with human chromosome-specific libraries: Detection of trisomy 21 and translocations of chromosome 4 . Proc Natl Acad Sci USA 85:9138-9141, 1988

18. Zhang J, Meltzer P, Jenkins R, Guan XY, Trent $J$ : Application of chromosome microdissection probes for elucidation of BCR-ABL fusion variant Philadelphia chromosome translocations in chronic myelogenous leukemia. Blood 81:3365-3371, 1993

19. Guan XY, Meltzer P, Trent J: Rapid generation of whole chromosome painting probes (WCPs) by chromosome microdissection. Genomics 22:101-107, 1994

20. Weber BL, Abel KJ, Couch FJ, Merajver S, Castilla L, Brody LC, Collins FS: Transcript identification in the BRCA1 candidate region. Breast Cancer Res Treat (this issue)

21. Hall J, Lee M, Morrow J, Newman B, Anderson L, Huey B, King M: Linkage of early-onset familial breast cancer to chromosome 17q21. Science 250: 1684-1689,1992

22. Abel K, Boehnke M, Prahalad M, Ho P, Flejter W, Watkins $M$, Vanderstoep J, Chandrasekharappa $S$, Collins F, Glover T, Weber B: A radiation hybrid map of the BRCA1 region of chromosome 17q12-q21. Genomics 17:632-641, 1993

23. Kallioniemi A, Kallioniemi OP, Waldman F, Chen L, Yu LC, Fung Smith H, Pinkel D, Gray J: Comparative genomic hybridization: A powerful new method for cytogenetic analysis of solid tumors. Science 258:818-820, 1991

24. Liang B, Meltzer P, Guan X-Y, Trent J: Genetic amplification elucidated by combined comparative genomic hybridization and chromosomal microdissection. Cancer Genet Cytogenet (in press) 